\title{
A BELIEF REVISION APPROACH FOR ARGUMENTATION-BASED NEGOTIATION AGENTS
}

\author{
PABlo PILOTTI $^{a, *}$, AnA CASAli $^{a}$, CARlos CHESÑEVAR $^{b}$ \\ ${ }^{a}$ Group of Fundamentals and Applications of Logic and Programming \\ International French-Argentinean Center for Systems and Information Sciences (CIFASIS) \\ Av. 27 de Febrero 210 bis, S2000EZP, Rosario, Argentina \\ e-mail: \{pilotti, casali\}@cifasis-conicet.gov.ar \\ ${ }^{b}$ Department of Computer Science and Engineering \\ National University of the South (UNS) \\ Av. Alem 1253 B8000CPB, Bahía Blanca, Argentina \\ e-mail: cicecs.uns.edu.ar
}

\begin{abstract}
Negotiation is an interaction that happens in multi-agent systems when agents have conflicting objectives and must look for an acceptable agreement. A typical negotiating situation involves two agents that cannot reach their goals by themselves because they do not have some resources they need or they do not know how to use them to reach their goals. Therefore, they must start a negotiation dialogue, taking also into account that they might have incomplete or wrong beliefs about the other agent's goals and resources. This article presents a negotiating agent model based on argumentation, which is used by the agents to reason on how to exchange resources and knowledge in order to achieve their goals. Agents that negotiate have incomplete beliefs about the others, so that the exchange of arguments gives them information that makes it possible to update their beliefs. In order to formalize their proposals in a negotiation setting, the agents must be able to generate, select and evaluate arguments associated with such offers, updating their mental state accordingly. In our approach, we will focus on an argumentation-based negotiation model between two cooperative agents. The arguments generation and interpretation process is based on belief change operations (expansions, contractions and revisions), and the selection process is a based on a strategy. This approach is presented through a high-level algorithm implemented in logic programming. We show various theoretical properties associated with this approach, which have been formalized and proved using Coq, a formal proof management system. We also illustrate, through a case study, the applicability of our approach in order to solve a slightly modified version of the well-known home improvement agents problem. Moreover, we present various simulations that allow assessing the impact of belief revision on the negotiation process.
\end{abstract}

Keywords: argumentation-based negotiation, collaborative agents, belief revision, multi-agent system.

\section{Introduction}

Negotiation is a form of interaction in which two or more agents with different goals find some acceptable agreement. A typical scenario for negotiation involves two agents which have the need to collaborate for mutual benefit. Even though there is no agreed approach to characterizing all negotiation frameworks, it has been argued (Jennings et al., 2001) that automated negotiation research can be considered to deal with three broad topics: negotiation protocols (the set of rules that govern the

\footnotetext{
*Corresponding author
}

interaction), negotiation objects (the range of issues over which agreement must be reached) and agents' decision making model (which accounts for the decision making apparatus the participants employ to act in line with the negotiation protocol in order to achieve their objectives).

Moreover, various approaches can be used to model negotiation in a multiagent setting (MAS). Rahwan et al. (2003) distinguish three different kinds of such approaches: those which are game-theoretic, those which are heuristic-based, and finally those based on argumentation (argumentation-based negotiation, or ABN for short). Game-theoretic approaches are built on studying 
and developing strategic negotiation models based on game-theory precedents (Rosenschein and Zlotkin, 1994). The interaction in the negotiation process is considered a game in which each agent tries to maximize its utility. Given a protocol, most researchers in this line of work attempt to analyze the optimal strategy. While this approach is very powerful in terms of analyzing the obtained results, it suffers from some drawbacks due to the assumptions upon which it is built. The most important ones are that agents are only allowed to exchange offers without any other information. On the other hand, heuristic-based approaches come to cope with some limitations of the game-theoretic approach. Some strong assumptions made in the latter are relaxed using heuristics. Most of these assumptions concern the notion of rationality of agents as well as their resources. The support for particular heuristics is usually based on empirical testing and evaluation. In general, these methods offer approximations to the decisions made according to game-theoretic studies. Unfortunately, most of those approaches assume that agents have unbounded computational resources and that the space of outcomes is completely known. In most realistic environments, however, these assumptions fail.

Finally, argumentation-based negotiation has been proposed as an alternative to the two previous approaches (Parsons et al., 1998; Rahwan et al., 2003; 2007; Amgoud and Vesic, 2011). This approach allows the negotiating agents not only to exchange offers but also reasons that support these offers in order to mutually influence their preference relation on the set of offers, and consequently the outcome of the dialogue. Moreover, as the agents that negotiate usually have incomplete beliefs about the others, the exchange of arguments gives them information that makes it possible to update their beliefs. Rahwan et al. (2003) depict an ABN framework in terms of the interaction between the negotiating agents and the environment. They outline those central elements in the design of an $\mathrm{ABN}$ framework, classifying them into external and internal (wrt the agent). External elements are those that define the environment in which $\mathrm{ABN}$ agents operate and interact: communication language (the language that facilitates the negotiation, normally including basic locutions such as propose, accept and reject), domain language (the language for referring to concepts of the environments, agents, resources, etc.); the negotiation protocol (the conventions that govern the interaction among participants), and information stores (stores that keep track of relevant information for the negotiation externally, such as past utterances, reputations of the participants, etc.). On the other hand, internal elements are the main components needed by an agent in order to be capable of engaging in a negotiation.

Figure 1 shows the sketch of a generic ABN agent, identifying the components discussed before, as well as their interrelationship. The locution interpretation component parses incoming messages. These locutions usually contain a proposal, or an acceptance or rejection message of a previous proposal. The proposal database component stores proposals for future reference. The proposal evaluation and generation component makes a decision about whether to accept, reject or generate a counterproposal, or even terminate the negotiation. The locution generation component sends the response to the relevant party. The argument interpretation component updates the agents' mental state accordingly. Finally, the argument generation mechanism is responsible for deciding what response to actually send to the counterpart and what (if any) arguments should accompany the response.

In order to formalize their offers in a negotiation setting, ABN agents must be able to generate, select and evaluate arguments associated with such offers, updating their mental state accordingly. In this paper, we focus on providing a novel characterization for these elements, proposing an argumentation-based negotiation model between two cooperative agents. For our analysis we will assume that each agent is benevolent (it will always try to do what is asked for if it is able to do so) and truthful (i.e., it will not knowingly communicate false information). Besides, we will assume that neither of the agents can reach their respective goals by themselves, so that they have to ask for help from one another. The agents can thus exchange different resources, including the knowledge associated with possible plans to reach their goals.

The resulting negotiation dialog is composed of an exchange of proposals, where every proposal adopts the form of an argument whose claim is a possible exchange (which are the resources the agent is asking for and what it is willing to offer in return). As the agents initially may have incomplete or wrong beliefs about the other agent's goals and resources, during the negotiation process they update their beliefs and, consequently, their mental state, according the arguments exchanged. Thus, in the context of the $\mathrm{ABN}$ framework previously described, we will use

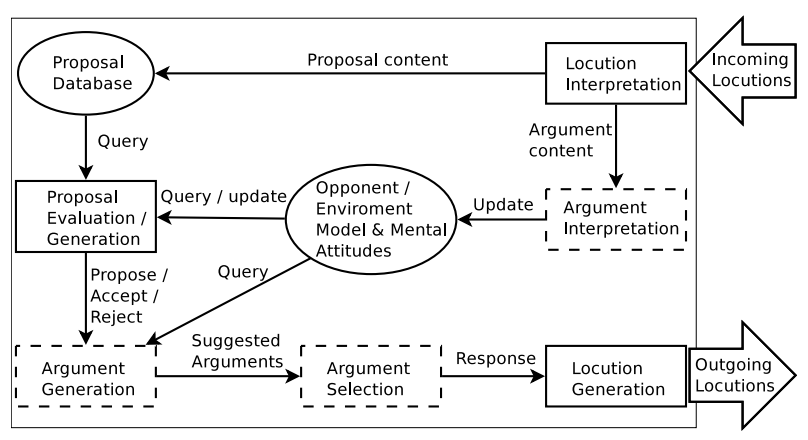

Fig. 1. Elements of an ABN agent (Rahwan et al., 2003) 
a belief revision approach for both argument interpretation and argument generation. The proposed approach is presented through a high-level algorithm implemented in logic programming. We will show different theoretical properties associated with this negotiation model, which have been formalized and proved using $\operatorname{Coc} 1$, a formal proof management system. We will also illustrate through a case study the applicability of our approach in order to solve a motivational example.

In order to carry out an empirical assessment of our proposal, simulations of different types of agents were run, considering 100 possible negotiating scenarios. For these simulations, three agent types are defined: (a) NBR agents, which do not make use of belief revision, (b) PBR agents, which make partial use of belief revision, and (c) BR agents, which apply belief revision on all the information contained in the received messages. Our experiments allowed us to identify relevant features and potential advantages of those negotiating agents that make full use of belief revision by considering all the information received from their counterpart in the negotiation process.

Motivational example. For the rest of this article, we will work on a slightly modified version of the well-known home improvement agents (HIA) problem as a motivational example (Parsons et al., 1998). We will assume two benevolent agents $A g_{1}$ and $A g_{2}$. Agent $A g_{1}$ has as the goal of hanging a picture, and it has a screw and a hammer. Also, it knows how a hammer and a nail can be used to hang a picture and how a screw and a screwdriver can be used to hang mirrors. $A g_{1}$ believes that $A g_{2}$ has a nail and a screwdriver (a correct, but incomplete belief), and it believes that $\mathrm{Ag}_{2}$ 's goal is to have a screw (wrong belief). On the other hand, Agent $\mathrm{Ag}_{2}$ has as a goal to hang a mirror, and it has a nail, a screwdriver and the knowledge of how to hang a mirror using a hammer and a nail. Neither $A g_{1}$ nor $A g_{2}$ can reach their goals on the basis of their knowledge and resources. Consequently, they need to perform some exchanges in order to do so. Our proposal aims at modelling how such exchanges can be determined by combining belief revision and argumentation.

The remainder of this paper is structured as follows. In Sections 2 and 3 we define the agent architecture and the negotiation protocol, formalizing the notions of proposal, dialogue and deal. Then in Section 4 we show how to integrate belief revision operators in a high-level algorithm for solving negotiation problems between two argumentative agents. We also discuss some theoretical properties of our approach. In Section 5 we show how the

\footnotetext{
${ }^{1} \mathrm{Coq}$ http://coq.inria.fr/) is an interactive theorem prover. It provides a formal language to write mathematical definitions, executable algorithms and theorems, together with an environment for semi-interactive development of machine-checked proofs.
}

HIA problem can be solved in the context of our proposal. Then, in Section 6, we present simulations of three types of agents in diverse negotiation scenarios where different advantages and salient features of agents using belief revision can be assessed. Section7 7 discusses related work, and finally in Section 8 we discuss the main conclusions obtained and outline some future research topics.

\section{Agent architecture}

For our negotiation scenario, each agent will have, in its mental state, resources, goals and plans, as well as beliefs on the other agent's resources and goals. From the information available in such a mental state, an agent will decide which proposals it can offer to the other agent in order to reach an agreement. In order to characterize the agent architecture, we will consider a propositional language $\mathcal{L}$, in which the following subsets are distinguished:

- Object $_{\mathcal{L}}$ : a set of atoms representing objects which are the resources an agent may have (e.g., nail, hammer);

- $G_{\mathcal{L}}:$ a set of atoms representing goals (e.g., hangMir represents the goal of hanging a mirror);

- Plans ${ }_{\mathcal{L}}$ : a set of propositional formulae encoding plans, which may involve objects for achieving a goal (e.g., nail $\wedge$ hammer $\rightarrow$ hangPict).

In several areas of computer science (e.g., operating systems), the term resources is generally considered in a broadest sense and can represent memory, programs, commodities, services, time, money, etc. In short, anything that is needed to achieve something. In this work the set of resources, noted by $R_{\mathcal{L}}$, will also include plans for achieving goals, i.e., $R_{\mathcal{L}}=$ Object $_{\mathcal{L}} \cup$ Plans $_{\mathcal{L}}$.

The plans represent the agent's knowledge of how to use objects to reach a particular goal. Consequently, a plan will be considered a special kind of resource that the agent can share with others without consuming it. We assume that an agent can have infinite copies of each plan it knows. Given a set $X \subset R_{\mathcal{L}}$, we will write $X^{\downarrow o}$ and $X^{\downarrow p}$ to distinguish the subset of objects and the subset of plans in $X$, respectively. Formally, $X^{\downarrow o}={ }_{\text {def }} X \cap$ Objects $_{\mathcal{L}}$ and $X^{\downarrow p}={ }_{\operatorname{def}} X \cap$ Plans $_{\mathcal{L}}$.

Definition 1. (Agent mental state) Let two agents $A g_{i}$, $A g_{j}$ be involved in a negotiation. The mental state (MS) of Agent $A g_{i}$ is the quintuple $M S_{i}=\left\langle R_{i}, G_{i}, B_{i} R_{j}, B_{i} G_{j}\right.$, $\left.H_{i}\right\rangle$, where $R_{i}, B_{i} R_{j} \subset R_{\mathcal{L}} ; G_{i}, B_{i} G_{j} \subset G_{\mathcal{L}}$ and $H_{i}$ is the history of the negotiation 2 .

\footnotetext{
${ }^{2}$ In what follows, we will refer to $A g_{i}$ as a generic agent, $A g_{j}$ being the counterpart agent.
} 
Thus, the mental state of $A g_{i}$ includes a set of available resources $\left(R_{i}\right)$ the agent is willing to negotiate, a set of goals to achieve $\left(G_{i}\right)$, as well as belief sets about which resources are available for the opponent Agent $A g_{j}\left(B_{i} R_{j}\right)$, and which goals it believes Agent $A g_{j}$ has $\left(B_{i} G_{j}\right)$. Its mental state includes as well the history of the dialogue (see Definition 6) with $A g_{j}$.

Example 1. Consider the HIA problem given in Section 1. In the beginning of the negotiation process, $A g_{1}$ 's mental state can be represented as $M S_{1}=$ $\left\langle R_{1}, G_{1}, B_{1} R_{2}, B_{1} G_{2}, H_{1}\right\rangle$, where

$$
\begin{aligned}
& R_{1}=\{\text { screw, hammer }, \\
& \text { screw } \wedge \text { screwDriver } \rightarrow \text { hangMir }, \\
&\text { hammer } \wedge \text { nail } \rightarrow \text { hangPict }\}, \\
& G_{1}=\{\text { hangPict }\}, \\
& B_{1} R_{2}=\{\text { nail, screwDriver }\}, \\
& B_{1} G_{2}=\{\text { screw }\}, \\
& H_{1}=[],
\end{aligned}
$$

and similarly, $A g_{2}$ 's mental state can be represented as $M S_{2}=\left\langle R_{2}, G_{2}, B_{2} R_{1}, B_{2} G_{1}, H_{2}\right\rangle$, where

$$
\begin{aligned}
R_{2}= & \{\text { hammer } \wedge \text { nail } \rightarrow \text { hangMir }, \\
& \text { nail, screwDriver }\}, \\
G_{2}= & \{\text { hangMir }\}, \\
B_{2} R_{1}= & \{\text { nail }\}, \\
B_{2} G_{1}= & \{\}, \\
H_{2}= & {[] . }
\end{aligned}
$$

From a global viewpoint we want to characterize the sets that account for the agents' correct, wrong and missing beliefs with respect to its counterparts resources. Formally, we have the following.

Definition 2. (Missing, correct and wrong beliefs) Let $A g_{i}, A g_{j}$ be two agents. We shall write $M_{i}$ to denote the set of resources that $A g_{i}$ does not know that $A g_{j}$ has, $T_{i}$ to denote the set of resources that $A g_{i}$ believes that $A g_{j}$ has and this is actually the case, i.e., such beliefs are correct, and $F_{i}$ to denote the set of resources that $A g_{i}$ believes that $A g_{j}$ has and this is actually not the case, i.e., such beliefs are wrong. Formally $M_{i}=\overline{B_{i} R_{j}} \cap R_{j}$, $T_{i}=B_{i} R_{j} \cap R_{j}$, and $F_{i}=B_{i} R_{j} \cap \overline{R_{j}}$.

Thus, in Example1Agent $A g_{1}$ believes that $A g_{2}$ has nail and screwDriver, and this is correct, so that $T_{1}=$ $\{$ nail, screwDriver $\}$ but it does not know that $A g_{2}$ also has the plan hammer $\wedge$ nail $\rightarrow$ hangMir, so that $M_{1}=$ $\{$ hammer $\wedge$ nail $\rightarrow$ hangMir $\}$. As $A g_{1}$ does not have wrong beliefs, in this particular case $F_{1}=\emptyset$, as shown in Fig. 2. Similarly, we can determine these sets for $\mathrm{Ag}_{2}$ as $M_{2}=\{$ screw, hammer, screw $\wedge$ screwDriver $\rightarrow$ hangMir, hammer $\wedge$ nail $\rightarrow$ hangPict $\}, T_{2}=\{\}$ and $F_{2}=\{$ nail $\}$.

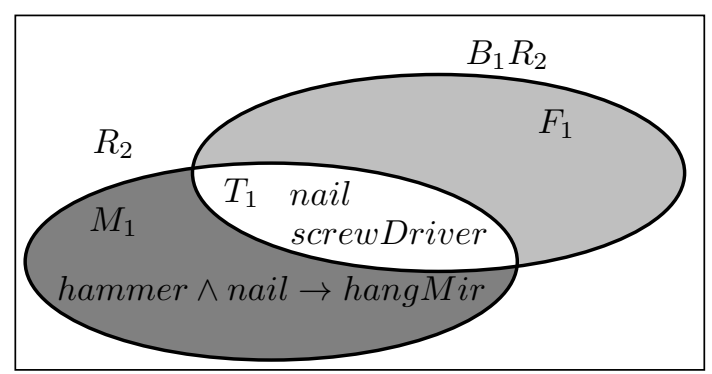

Fig. 2. $M_{1}$ : missing beliefs (dark gray), $T_{1}$ : correct beliefs (white), and $F_{1}$ : wrong beliefs (gray).

The decision making apparatus the agents employ to act in order to achieve their objectives depends on their mental states (see Definition 1). This apparatus will be in charge of computing those proposals the agent will make to the other agent. We will write Proposal to denote the set of all possible proposals (the formal definition of a proposal is given in Definition 5). As the first dialogue move associated with the initial proposal is a particular one, we will single it out by using an initialization function Init. Further proposals and counter-proposals are computed by another function Answer. Formally, we have the following.

Definition 3. (Decision making apparatus) The decision making apparatus of Agent $A g_{i}$ is a tuple $D M_{i}=$ $\left\langle\right.$ Init $_{i}$, Answer $\left._{i}\right\rangle$, where

$$
\begin{aligned}
& \text { Init }_{i}: M S_{i} \rightarrow M S_{i} \times \text { Proposal, } \\
& \text { Answer } i: M S_{i} \times \text { Proposal } \rightarrow M S_{i} \times \text { Proposal } .
\end{aligned}
$$

We will purposely leave unspecified the actual definitions of Init $_{i}$ and Answer $_{i}$ at this stage. Later on, in Section 4, we will provide their specification through high-level algorithms. Thus, in our approach, an ABN agent model will be composed of its mental state and its decision making apparatus. Formally, we have the following.

Definition 4. (Agent model) Agent $A g_{i}$ is the pair $\left\langle M S_{i}, D M_{i}\right\rangle$, where $M S_{i}$ is its mental state and $D M_{i}$ its decision making apparatus.

\section{Generating proposals as arguments to reach deals}

Based on their mental states, the agents using their decision apparatus will generate proposals towards reaching their goals. In our formalization, a proposal is 
a statement that includes what the agent wants to obtain and what the agent is willing to give in return, together with an explanation justifying why an agent needs what it is asking for. Thus, proposals will have the following intended meaning:

I propose that you provide me with $Y$ in exchange of $X$, because if I use $O$, then I can achieve $G$,

where $Y, O$, and $X$ stand for resources, and $G$ is a set of goals.

Note that an agent's proposal can be thought of as an argumen 3 wose claim is associated with what the agent needs to achieve its goals (namely, $Y$ ) and the resources that the agent offers in exchange $(X)$, together with its support, i.e., the reasons given for requesting that resource from the other agent. The following definition formalizes this concept.

Definition 5. (Proposal) Let $X, Y, O$ be subsets of $R_{\mathcal{L}}$, and let $G$ be a subset of $G_{\mathcal{L}}$. A proposal performed by $A g_{i}$ is the pair $\left\langle\mathcal{A}, \llbracket X, Y \rrbracket_{i}\right\rangle$, where $\llbracket X, Y \rrbracket_{i}$ corresponds to the claim of the argument, and $\mathcal{A}=(O, G)$ provides the support associated with the claim, and the following conditions hold:

$$
\begin{array}{r}
Y \cup O \vdash G, \\
O \nvdash G, \\
X \cap(Y \cup O)=\emptyset .
\end{array}
$$

A proposal $\left\langle\mathcal{A}, \llbracket X, Y \rrbracket_{i}\right\rangle$ will be minimal iff there is no other proposal $\left\langle\mathcal{A}, \llbracket X, Y^{\prime} \rrbracket_{i}\right\rangle$, such that $Y^{\prime} \subset Y$.

Notice that (1) states that both the sets of resources $O$ and $Y$ are needed for the agent to reach the goal $G,(2)$ means the agent cannot reach the goal using only $O$ and (3) states that no element of $X$ is needed by the agent to reach $G$ as it suffices to use $Y \cup O$ to reach $G$, as stated in the condition (1) 4

Example 2. (Continuation of Example 1) Suppose that in this scenario $\mathrm{Ag}_{2}$ begins the negotiation process by offering $A g_{1}$ the following proposal:

I propose that you provide me with a hammer in exchange for nothing, because if I use a nail and the knowledge about how to hang a mirror using a nail and a hammer, then I can hang a mirror.

\footnotetext{
${ }^{3} \mathrm{~A}$ full account of argumentation theory as well as its applications in multiagent systems and belief revision is outside the scope of this article. For further references and insights, the reader is referred to Dix et al. (2013) and Falappa et al. (2011).

${ }^{4}$ We write $X \vdash G$ whenever $G \subseteq C n(X)$, where $C n$ is a logical consequence operator.
}

Then this proposal is denoted by $\left\langle\mathcal{A}, \llbracket\{\},\{\right.$ hammer $\left.\} \rrbracket_{2}\right\rangle$ where the support associated with the claim is $\mathcal{A}=$ $(\{$ nail, nail $\wedge$ hammer $\rightarrow$ hangMir $\},\{$ hangMir $\})$.

A dialogue between two agents will be defined as a finite sequence of proposals (which account for arguments in favor of some particular exchange), performed alternatively by each of the agents involved in the dialogue, ending with accept (there is a deal) or withdraw (no deal is possible).

Definition 6. (Negotiation dialogue) A dialogue between Agents $A g_{i}$ and $A g_{j}$ is a finite sequence of utterances $\left[u_{1}, \ldots, u_{n-1}, u_{n}\right]$ where, for $r<n, u_{r}$ is a proposal and $u_{n} \in\{$ accept, withdraw $\}$, such that (i) there are no repeated utterances, i.e., $u_{s} \neq u_{t}$, with $t, s<n$; (ii) utterance $u_{k}$ with $k>1$ is performed by Agent $A g_{i}$ only if utterance $u_{k-1}$ is performed by Agent $A g_{j}$ (i.e., agents alternate moves). A dialogue will be initiated by $A g_{i}$ iff $u_{1}$ is performed by $A g_{i}$.

Note that dialogues can be warranted to be finite, as there is a finite set of possible combinations of proposals and utterance repetition is not allowed. From Definitions 3 and 6 we can see that the dialogue between Agents $A g_{i}$ and $A g_{j}$ will be started by one of the agents with a proposal computed by Init, followed by a counter-proposal by the other agent computed by Answer, a counter-counter-proposal by the first agent, and so on. With no loss of generality we assume Agent $A g_{i}$ is the one who starts the negotiation dialogue. Figure 3 represents the negotiation dialogue flow initiated by $A g_{i}$ as a finite-state machine.

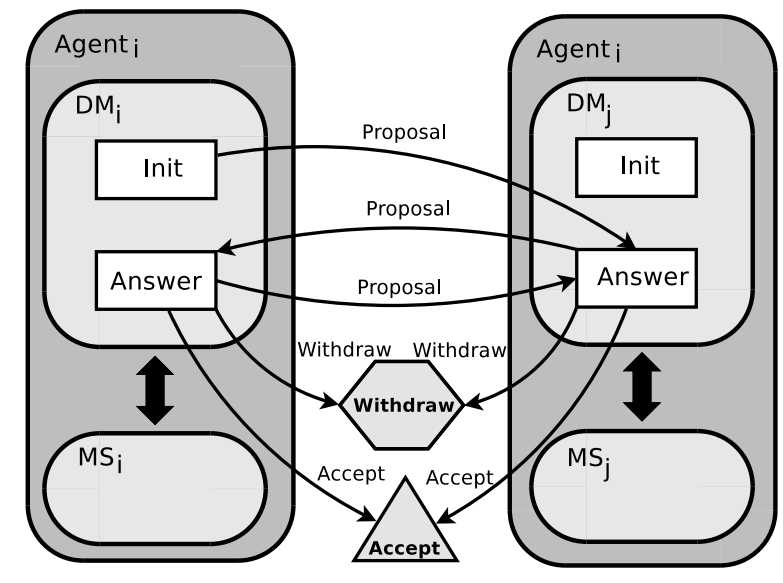

Fig. 3. Dialogue flow initiated by $A g_{i}$.

Proposal evaluation. As previously mentioned, we assume Agents $A g_{i}$ and $A g_{j}$ cannot reach their goals on their own, and, therefore the problem each agent faces is to find a suitable exchange of resources in the space of 
possible exchanges $\left(\mathcal{P}\left(R_{i}\right) \times \mathcal{P}\left(R_{j}\right)\right)$ in order to reach its own goal. In this setting, a proposal can be thought of as an argument $\left\langle(O, G), \llbracket X, Y \rrbracket_{i}\right\rangle$ supporting an exchange of resources. By definition, the pair of resources $\llbracket X, Y \rrbracket_{i}$ provides a solution to reach $A g_{i}$ 's goal.

We define the function $\odot$ that assigns to each proposal $\left\langle(O, G), \llbracket X, Y \rrbracket_{i}\right\rangle$ its associated solution 5

Following Rahwan et al. (2003), we assume that in our approach agents have an objective consideration when they evaluate proposals (i.e., they consider a proposal a tentative proof to reach their goals, and they verify it by examining the validity of its underlying assumptions, such as resource availability). Since each agent is aware of its own resources and goals, it can determine first, in a selfish way, which are the exchanges that provide a solution for its problem. This is formalized in the following definition.

Definition 7. Let $A g_{i}$ be an agent involved in a negotiation, where its mental state is $M S_{i}=$ $\left\langle R_{i}, G_{i}, B_{i} R_{j}, B_{i} G_{j}, H_{i}\right\rangle$. A solution for $A g_{i}$ is any pair $\llbracket X, Y \rrbracket_{i}, X, Y \subseteq R_{\mathcal{L}}$ such that

(i) $X \subseteq R_{i}$;

(ii) $\left(R_{i}-X^{\downarrow o}\right) \cup Y \vdash G_{i}$. for $A g_{i}$.

We will denote by $\mathcal{S}_{i}$ the set of all possible solutions

Note that $X$ stands for those resources that $A g_{i}$ is willing to give to $A g_{j}$, whereas $Y$ is the set of resources that are given to $A g_{i}$ to achieve its goal. In a similar way $\mathcal{S}_{j}$ is defined. A deal for $A g_{i}$ and $A g_{j}$ will be a solution which is applicable for both of them, being formally defined as follows.

Definition 8. We will say that $\llbracket X, Y \rrbracket_{i}$ where $X, Y \subseteq R_{\mathcal{L}}$, is a deal for $A g_{i}$ and $A g_{j}$ iff $\llbracket X, Y \rrbracket_{i} \in \mathcal{S}_{i} \wedge \llbracket Y, X \rrbracket_{j} \in \mathcal{S}_{j}$. We will denote by $\mathcal{D}$ the set of all deals between $A g_{i}$ and $A g_{j}$.

From the definitions presented before, the agents' evaluation process can be defined in a simple way as follows: If prop $=\left\langle(O, G), \llbracket X, Y \rrbracket_{i}\right\rangle$ is an $A g_{i}$ proposal, then prop will be accepted by $A g_{j}$ if $\llbracket Y, X \rrbracket_{j} \in \mathcal{S}_{j}$. Notice that a proposal prop will be accepted only if it is a deal.

\section{Integrating belief revision in $\mathrm{ABN}$ agents}

In this section we will show how belief revision can be used in an ABN agent to improve two important issues in a negotiation: (i) proposal interpretation and (ii) proposal generation. We assume that the information contained in a proposal can be used by an agent to revise the beliefs it has about the other agent and then, by having more accurate beliefs, the first agent can make proposals that are

\footnotetext{
${ }^{5}$ The function $\odot$ corresponds to the second component projection.
}

more likely to be accepted. In order to make our analysis self-contained, we will summarize some notions of belief change theory that will be applied in our approach.

4.1. Belief revision operators. Classic belief change operations introduced in the AGM model (Alchourrón et al., 1985) are known as expansions, contractions and revisions. An expansion incorporates a new belief without warranting the consistency of the resulting epistemic state. A contraction eliminates a belief $\alpha$ from the epistemic state as well as all those beliefs that make the inference of $\alpha$ possible. Finally, a revision incorporates a new belief $\alpha$ to the epistemic state warranting a consistent result, assuming that $\alpha$ itself is consistent.

As discussed before, in our setting we assume that the agents have their own beliefs about the other agent's resources and goals. It must be noted that the sets of resources and objectives do not change during the negotiation. Only if a deal succeeds at the end of the negotiation process will the actual exchange of resources take place, and consequently the sets $X$ and $Y$ will be changed. In order to model such a negotiation process in terms of belief revision, we will use the notion of the choice kernel set and multiple choice contraction proposed by Hansson (1994) and followed by Fermé $e t$ al . (2003). These notions will be useful for providing a practical approach to belief revision in our context.

We provide below a brief review of the formal definitions involved.

Definition 9. (Choice kernel set) (Fermé et al., 2003) Let $\mathcal{L}$ be a logical language, $C n$ a consequence operator, $R \subseteq \mathcal{L}$ and $G \in \mathcal{L}$. Then $R \Perp G$ is the set of all $X \subseteq R$ such that

(i) $G \subseteq C n(X)$;

(ii) If $Y \subset X$, then $G \nsubseteq C n(Y)$.

The set $R \Perp G$ is called the choice kernel set, and its elements are called $G$-kernels of $R$.

Informally, a choice kernel set is a minimal belief subset of the epistemic state from which $G$ can be deduced. An element in $R$ contributes to make $R$ imply $G$ if and only if it is an element of some $G$-kernels of $R$. Therefore, removing at least one element of each $G$-kernels of $R$, it is no longer possible to derive $G$. The function that selects sentences to be removed will be called an incision function since it makes an incision into every $G$-kernel.

Definition 10. (Incision function) (Fermé et al., 2003) A function $\sigma$ is an incision function for $R$, iff it satisfies, for all $G$,

(i) $\sigma(R \Perp G) \subseteq \bigcup(R \Perp G)$;

(ii) If $\emptyset \neq X \in R \Perp G$, then $X \cap \sigma(R \Perp G) \neq \emptyset$. 
The multiple choice contraction operator allows to remove the elements selected by an incision function. Formally:

Definition 11. (Multiple choice contraction) (Fermé et al., 2003) Let $\sigma$ be an incision function for $R$ and $G \in \mathcal{L}$. The multiple choice contraction $\approx$ for $R$ is defined as

$$
R \approx G=R-\sigma(R \Perp G) .
$$

Next, a revision operator is expressed using two sub-operations: first a contraction and then an expansion (i.e., adding $G$ to the resulting set).

Definition 12. (Revision operator) (Hansson, 1999) Let $\approx$ be a global kernel contraction. Given a set of sentences $R$, we define for any set $G$ the revision operator $*: R * G=$ $(R \approx \neg G) \cup G$

Contracting by the finite set $\neg G$ is equivalent to contracting by a single formula, namely, the disjunction of all negations of elements in $G$.

4.2. Argument generation. In a negotiation dialogue, the beliefs a particular agent has about the other agent's resources and goals are significant for proposal generation, as they can help reaching a deal. From this information, an agent can infer which proposals it believes are more suitable for the other and, consequently, more likely to be accepted. To formalize this notion, we define the following concepts.

Definition 13. Let $A g_{i}$ and $A g_{j}$ be two agents and $X, Y \subseteq$ $R_{\mathcal{L}}$. We will say that $A g_{i}$ believes $\llbracket X, Y \rrbracket_{i}$ is a solution for $A g_{j}$ whenever

(i) $Y \subseteq B_{i} R_{j}$;

(ii) $\left(B_{i} R_{j}-Y^{\downarrow o}\right) \cup X \vdash B_{i} G_{j}$.

$$
\begin{aligned}
& \text { Define } \\
& \mathcal{B}_{i} \mathcal{S}_{j}=\left\{\llbracket X, Y \rrbracket_{i} \mid\right.
\end{aligned}
$$

$A g_{i}$ believes $\llbracket X, Y \rrbracket_{i}$ is a solution for $\left.A g_{j}\right\}$.

Definition 14. Let $A g_{i}$ and $A g_{j}$ be two agents. We will say that $A g_{i}$ believes $\llbracket X, Y \rrbracket_{i}$ is a deal iff

(i) $X \subseteq R_{i}$;

(ii) $\left(R_{i}-X^{\downarrow o}\right) \cup Y \vdash G_{i}$;

(iii) $Y \subseteq B_{i} R_{j}$;

(iv) $\left(B_{i} R_{j}-Y^{\downarrow o}\right) \cup X \vdash B_{i} G_{j}$.

Define

$\mathcal{B}_{i} \mathcal{D}=\left\{\llbracket X, Y \rrbracket \mid A g_{i}\right.$ believes $\llbracket X, Y \rrbracket_{i}$ is a deal $\}$. hold 6

From Definitions 13 and 14 the following results

Proposition 1. $\llbracket X, Y \rrbracket_{i} \in \mathcal{S}_{i}$ and $\llbracket X, Y \rrbracket_{i} \in \mathcal{B}_{i} \mathcal{S}_{j} \Leftrightarrow$ $\llbracket X, Y \rrbracket_{i} \in \mathcal{B}_{i} \mathcal{D}$.

Proposition 2. $\llbracket X, Y \rrbracket_{i} \in \mathcal{B}_{i} \mathcal{D}$ and $\llbracket Y, X \rrbracket_{j} \in \mathcal{S}_{j} \Rightarrow$ $\llbracket X, Y \rrbracket_{i} \in \mathcal{D}$.

Proposition 3. $\llbracket X, Y \rrbracket_{i} \in \mathcal{B}_{i} \mathcal{D}$ and $\llbracket Y, X \rrbracket_{j} \in \mathcal{B}_{j} \mathcal{D} \Rightarrow$ $\llbracket X, Y \rrbracket_{i} \in \mathcal{D}$.

Proposition 1 states that if a pair $\llbracket X, Y \rrbracket_{i}$ is a solution for $A g_{i}$ and it believes that it is also a solution for $A g_{j}$, then $A g_{i}$ believes that $\llbracket X, Y \rrbracket_{i}$ is a deal, and the reciprocal is also held. Similarly, Proposition 2 asserts that if Agent $A g_{i}$ believes that $\llbracket X, Y \rrbracket_{i}$ is a deal and $\llbracket Y, X \rrbracket_{j}$ is also a solution for $A g_{j}$, then $\llbracket X, Y \rrbracket_{i}$ is a deal. Finally, Proposition 3 states that if $A g_{i}$ believes that $\llbracket X, Y \rrbracket_{i}$ is a deal and $A g_{j}$ believes that $\llbracket Y, X \rrbracket_{j}$ is a deal, then it holds that $\llbracket X, Y \rrbracket_{i}$ is a deal.

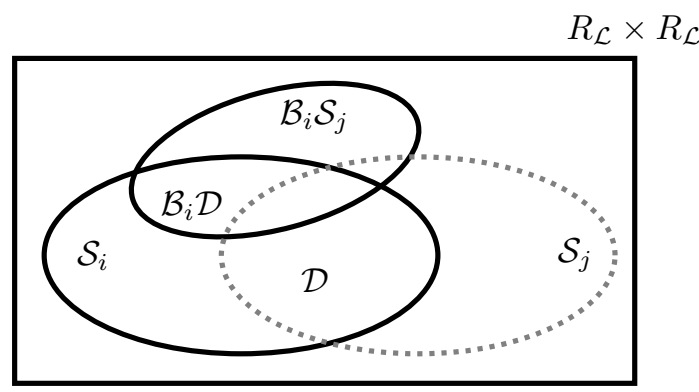

Fig. 4. Solutions' space from the $A g_{i}$ viewpoint.

Figure 4 shows the set of solutions from the viewpoint of $A g_{i}$. The dotted line represents that the agent does not know $\mathcal{S}_{j}$. Because of this, $A g_{i}$ cannot be sure of making a proposal prop such that $\odot($ prop $) \in \mathcal{D}$. So, in order to entice Agent $A g_{j}$ to accept some proposed agreement, $A g_{i}$ must choose a proposal prop such that it believes its associated solution is a deal, i.e., $\odot($ prop $) \in$ $\mathcal{B}_{i} \mathcal{D}$.

The function Gen is defined to compute the proposals that are a solution to $A g_{i}$ (i.e., $\odot($ prop $) \in \mathcal{S}_{i}$ ) and to compute proposals that are potential solutions (i.e., $\odot($ prop $\left.) \in \mathcal{B}_{i} \mathcal{S}_{j}\right)$. The Gen function is specified using belief revision operations, and some properties that follow from its specification are given.

Definition 15. Let $R, R^{\prime} \subset R_{\mathcal{L}}$ and $G \subset G_{\mathcal{L}}$. We define a function Gen as

\footnotetext{
${ }^{6}$ All the propositions and their proofs were formalized in Coq and are available at http://web.cifasis-conicet.gov.ar/ pilotti/Automated_Agent_Negotiation.v
} 


$$
\begin{aligned}
\operatorname{Gen}(R, & \left.R^{\prime}, G, i\right) \\
\stackrel{\text { def }}{=} & \left\{\left\langle(O, G), \llbracket X, Y \rrbracket_{i}\right\rangle: Y \cap R=\emptyset, O \subseteq R,\right. \\
& (O \cup Y) \in\left(R \cup R^{\prime} \cup Y\right) \Perp G, \\
& X \subseteq R-O\} .
\end{aligned}
$$

In Definition 15 the Gen function receives two sets of resources $\left(R\right.$ and $R^{\prime}$ ) and a set of goals $(G)$. As an outcome it generates a set of proposals prop $=$ $\left\langle(O, G), \llbracket X, Y \rrbracket_{i}\right\rangle$, where $Y$ and the first set of resources $(R)$ are disjoint sets, but $O$ is a subset of it. The union of $Y$ and $O$ is a minimal set from which $G$ can be deduced. The set $X$ corresponds to the unused resources of $R$ to achieve $G$.

Proposition 4. Given Agent $A g_{i}$, where its mental state is $M S_{i}=\left\langle R_{i}, G_{i}, B_{i} R_{j}, B_{i} G_{j}, H_{i}\right\rangle$, the following holds:

(i) If prop $\in \operatorname{Gen}\left(R_{i}, B_{i} R_{j}, G_{i}, i\right)$, then prop $\in$ Proposal and $\odot($ prop $) \in \mathcal{S}_{i}$;

(ii) If prop $\in \operatorname{Gen}\left(B_{i} R_{j}, R_{i}, B_{i} G_{j}, j\right)$, then prop $\in$ Proposal and $\odot($ prop $) \in \mathcal{B}_{i} \mathcal{S}_{j}$.

The condition (i) in Proposition 4 establishes that the Gen function computes all the minimal proposals that are solutions for $A g_{i}$ from its point of view, namely, using as parameters its resources $\left(R_{i}\right)$, its belief about the other agent's resources $\left(B_{i} R_{j}\right)$ and its goal $\left(G_{i}\right)$. On the other hand, in (ii) the Gen function computes the proposals that $A g_{i}$ thinks that are solutions for $A g_{j}$, i.e., using as parameters its beliefs about the other, agent's resources $\left(B_{i} R_{j}\right)$, its own resources $\left(R_{i}\right)$ and its belief about the other agent's goal $\left(B_{i} G_{j}\right)$. In summary, Proposition 4 shows that the possible proposals that can be generated via an implementation of Gen are potential solutions for the negotiation problem between the agents involved.

4.3. Argument selection. An important point related to the argument generation mechanism is argument selection. This mechanism should provide an answer to the following question: Given a set of proposals that an agent may send to its counterpart, which is the more appropriate from the point of view of the speaker?

Rahwan et al. (2003) present an overview of some relevant selection mechanisms - we summarize some of them. In the work of Kraus et al. (1998), arguments are selected according to strength order, from "appeal to prevailing practice" to "a threat". The intuition is that a negotiator would progress from weak arguments up to the strongest. In the framework by Ramchurn et al. (2003), agents use a fuzzy rules based system that combines trust and utility in order to decide which candidate argument to send with a request. Agents in the framework presented by Parsons et al. (1998) provide the strongest argument possible based on the acceptability classes (e.g., a tautological argument if possible). For Amgoud et al.
(2000), agents compare arguments based on preferential ordering over their constituent propositions in a manner similar to that in argument evaluation (i.e., based on the argumentation system of Dung (1995)). In the work of Sadri et al. (2001), agents can compare the costs of different alternative plans to present to the counterpart.

Sierra and Debenham (2007) consider five dimensions relevant to negotiations: legitimacy, options, goals, independence, and commitment. They introduce a negotiation model built upon an information-based measure (to represent the information gain) and a utility-based function (to represent the utility gain) defined for each one of these dimensions. The negotiation strategies rely on two primitive concepts: intimacy (degree of closeness) and balance (degree of fairness). Arguments are selected in order to obtain a successful deal and to reach a target intimacy level.

In our approach, inspired by Sierra and Debenham (2007) and considering only the dimension options (i.e., "the possible agreement the agent can accept"), the agent selection mechanism is based on an information function $I: H \times$ Proposal $\rightarrow \mathbb{R}$ (where $H$ stands for the history of the negotiation, see Definition 1) and a utility function $U$ : Proposal $\rightarrow \mathbb{R}$. Various selection mechanisms can be defined combining these functions to represent different agent behaviors. According to the agent personality and the relation it has with its counterpart agent (the intimacy relation), the function combining $I$ and $U$ may be defined in a suitable way (e.g., using a weighted sum).

4.4. Argument interpretation. When an agent receives a proposal, an argument interpretation mechanism must be invoked in order to update the agent's mental state accordingly. In our framework, the proposal interpretation is based on the following intuition: Since agents are truthful, benevolent and aware of their own resources, when Agent $A g_{j}$ receives a proposal prop $=\left\langle(O, G), \llbracket X, Y \rrbracket_{i}\right\rangle$ from $A g_{i}$, then $A g_{j}$ can infer the following information:

(i) If $A g_{i}$ asks for $Y$, then $A g_{j}$ believes $A g_{i}$ does not have $Y$ as resource;

(ii) If $A g_{i}$ uses $O$, then $A g_{j}$ believes $A g_{i}$ has $O$ as a resource;

(iii) If $A g_{i}$ offers $X$, then $A g_{j}$ believes $A g_{i}$ has $X$ as resource;

(iv) If $A g_{i}$ wants to reach $G$, then $A g_{j}$ believes $A g_{i}$ has $G$ as Goal.

Thus, $A g_{j}$ can change its beliefs accordingly, contracting its belief set as in 1 or revising it as in (ii)-(iv). In this way the computation of the belief set $\mathcal{B}_{i} \mathcal{S}_{j}$ may be closer to $\mathcal{S}_{j}$ and, consequently, the resulting set of possible deals $\mathcal{B}_{i} \mathcal{D}$ may be closer to $\mathcal{D}$ as well (as illustrated in Fig. 4). 
The agents will change their beliefs according to the intuitions presented before, using belief revision operations. Let contract and revise be implementations of the operators $\approx$ and $*$, respectively (see Definitions 11 and 12), and prop $=\left\langle(O, G), \llbracket X, Y \rrbracket_{i}\right\rangle$ an $A g_{i}$ proposal received by $A g_{j}$. The following steps, which can be seen as variable assignments, implement the agent's interpretation process:

(i) $B_{j} R_{i} \leftarrow \operatorname{contract}\left(B_{j} R_{i}, Y\right)$,

(ii) $B_{j} R_{i} \leftarrow \operatorname{revise}\left(B_{j} R_{i}, O\right)$,

(iii) $B_{j} R_{i} \leftarrow \operatorname{revise}\left(B_{j} R_{i}, X\right)$,

(iv) $B_{j} G_{i} \leftarrow \operatorname{revise}\left(B_{j} G_{i}, G\right)$.

4.5. Decision model: High-level algorithms. The agent's decision making apparatus has been defined in Section 2 and implemented using two algorithms Init and Answer. The algorithm Init is in charge of starting the negotiation. In a first place, it selects a proposal that Agent $A g_{i}$ believes is a deal $\left(\mathcal{B}_{i} \mathcal{D}\right)$ that has not been proposed before. If such a proposal does not exist, it tries to send a proposal associated with its own solutions $\left(\mathcal{S}_{i}\right)$. If this fails, the agent sends a withdraw message. On its turn, Answer receives the proposal generated from Init and checks if it is an associated solution to the agents problem, and in that case the proposal is accepted. If that is not the case, the agent's beliefs are revised and Init is invoked to generate a new proposal. High-level algorithms for Init $_{i}$ and Answer $_{i}$ are given next.

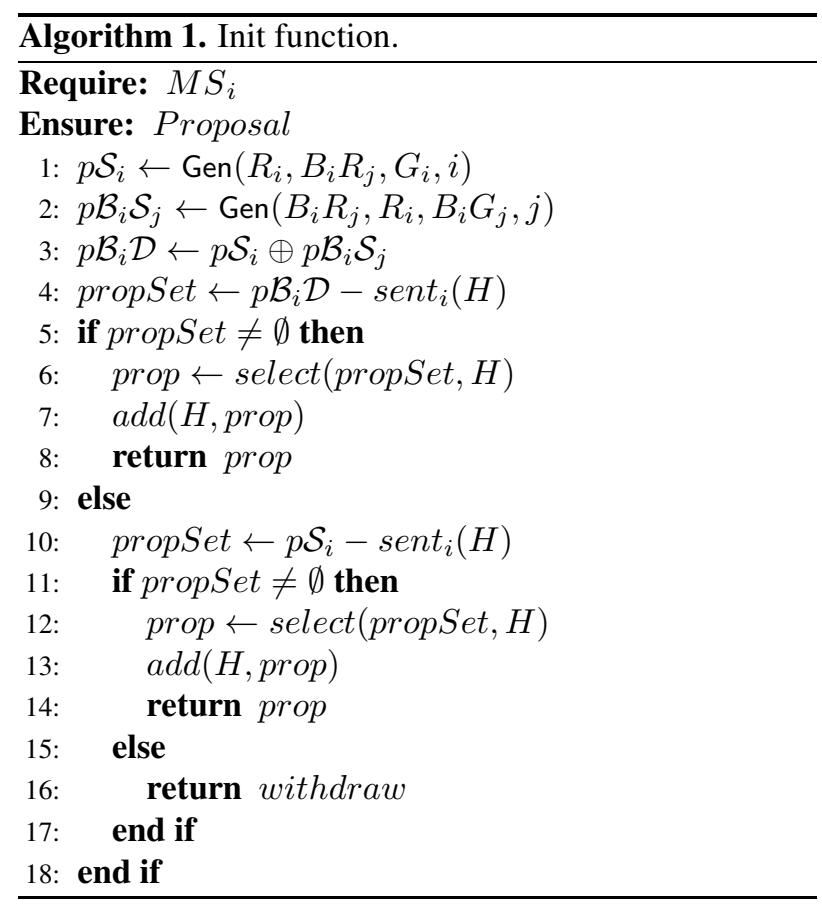

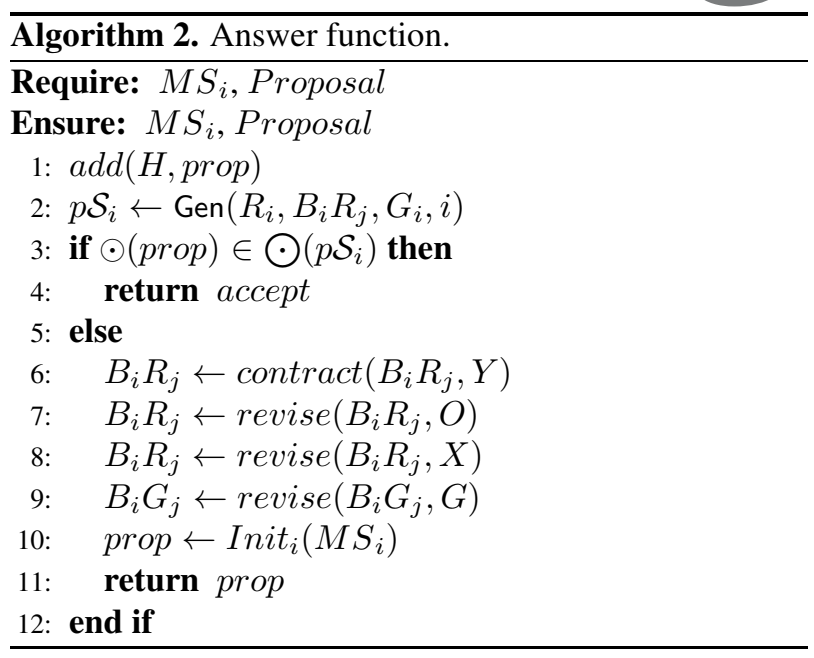

Algorithm 1: In Line 1, the function Gen (i.e., a suitable implementation of the Gen function specified in Definition 15) is used to compute the set of proposals $p \mathcal{S}_{i}$ such that their associated solutions belong to $\mathcal{S}_{i}$ (see Proposition 4). Similarly, in Line 2, Gen is used to compute the set of proposals $p \mathcal{B}_{i} \mathcal{S}_{j}$ that the agent believes their associated solutions belong to $\mathcal{B}_{i} \mathcal{S}_{j}$ (see Proposition 4). In Line 3, the set $p \mathcal{B}_{i} \mathcal{D}$ is computed as those proposals in $p \mathcal{S}_{i}$ such that their associated solutions are potential deals (see Proposition 1). In Line 4, those proposals that have been offered before are discarded. The select function chooses one proposal out of the set propSet of possible candidate proposals 7 Finally, the selected prop is added to $H$.

Algorithm 2: In Lines 1 and 2, the history $H$ is updated, and the set $p \mathcal{S}_{i}$ is computed. In Line 3, we check if the solution associated with the received proposal is a solution for $A g_{i}$. For this purpose, we use $\odot$ to denote the associated solution with a given proposal and $\odot$ to denote the set of associated solutions for a set of proposals. Then, in Lines 6 to 9, the agent updates its mental state following the steps presented in Section 4 For generating a counter-proposal the same lines of the code as the ones in Init are to be executed (as Init generates proposals). Therefore, for the sake of simplicity and in order to avoid repeating code, a call to Init is used in Line 10.

The proposed argumentation-based negotiation framework for two agents has been implemented using logic programming following the algorithms presented above. Based on such algorithms, concrete negotiating agents can be specified by instantiating their mental state and setting the selection function in charge to choose the proposal to negotiate.

\footnotetext{
${ }^{7}$ In Section 5, for the home improvement agents problem, we give an example of how this selection function may be defined.
} 


\section{HIA problem revisited}

As already mentioned in Section 1, we consider a slightly modified version of the home improvement agents example (Parsons et al., 1998) as a case study of our approach. We will assume two benevolent agents $A g_{1}$ and $A g_{2}$. Agent $A g_{1}$ has as goal hanging a picture, and it has a screw and a hammer. Also, it knows how a hammer and a nail can be used to hang a picture and how a screw and a screwdriver can be used to hang mirrors. $A g_{1}$ believes that $A g_{2}$ has a nail and a screwdriver (correct, but incomplete belief) and it believes that $A g_{2}$ 's goal is to have a screw (wrong belief). On the other hand, Agent $A g_{2}$ has as goal to hang a mirror, and it has a nail, a screwdriver and the knowledge of how to hang a mirror using a hammer and a nail. Therefore, $A g_{1}$ has the following initial mental state:

$$
\begin{aligned}
R_{1}= & \{\text { screw, hammer }, \\
& \text { screw } \wedge \text { screwDriver } \rightarrow \text { hangMir }, \\
& \text { hammer } \wedge \text { nail } \rightarrow \text { hangPict }\}, \\
G_{1}= & \{\text { hangPict }\}, \\
B_{1} R_{2}= & \{\text { nail, screwDriver }\}, \\
B_{1} G_{2}= & \{\text { screw }\}, \\
H_{1}= & {[], }
\end{aligned}
$$

and $A g_{2}$ has as its initial mental state

$$
\begin{aligned}
R_{2}= & \{\text { hammer } \wedge \text { nail } \rightarrow \text { hangMir }, \\
& \text { nail, screwDriver }\}, \\
G_{2}= & \{\text { hangMir }\}, \\
B_{2} R_{1}= & \{\text { nail }\}, \\
B_{2} G_{1}= & \{\}, \\
H_{2}= & {[] . }
\end{aligned}
$$

In this example, we assume that the agents select the proposal prop $\in$ Proposal that maximizes a weighted sum $\lambda_{U} U($ prop $)+\lambda_{I} I($ prop $)$. For simplicity, both agents use the same utility and information functions, but each agent considers different weights, which stand for different kinds of agents. For $A g_{1}$, the weights are $\lambda_{U}=$ $0.25, \lambda_{I}=2$, prioritizing the proposals that are more informative, and for $A g_{2}: \lambda_{U}=2, \lambda_{I}=0.25$, preferring those proposals that have a higher utility function. Also, we assume that the different resources they negotiate have the same costs for them. The agents' Utility function is defined as the difference of the cost of the resources offered to be exchanged, and it is expressed as follows:

$$
U_{i}(\text { prop })=\sum_{r \in Y} \operatorname{Cost}(r)-\sum_{r \in X} \operatorname{Cost}(r),
$$

where $\operatorname{prop}=\left\langle(O, G), \llbracket X, Y \rrbracket_{i}\right\rangle$ and

$$
\begin{aligned}
\text { Cost }= & \{(\text { hangMir }, 10),(\text { hangPict }, 10), \\
& (\text { hammer }, 4),(\text { screwDriver }, 4),(\text { screw }, 2), \\
& (\text { nail }, 2),(\text { hammer } \wedge \text { nail } \rightarrow \text { hangPict }, 8), \\
& (\text { screw } \wedge \text { screwDriver } \rightarrow \text { hangMir }, 8)\},
\end{aligned}
$$

and the agents' information function is defined as

$$
\begin{aligned}
I_{i}(H, \text { prop })= & \sum_{r \in Y} \mathbf{1}_{\text {get }}(H, r)+\sum_{r \in X} \mathbf{1}_{\text {give }}(H, r) \\
& +\sum_{r \in O} \mathbf{1}_{\text {own }}(H, r)+\sum_{r \in G} \mathbf{1}_{\text {goal }}(H, r),
\end{aligned}
$$

where $\mathbf{1}_{g e t}(H, r)$ returns 1 if, for all $\left\langle(O, G), \llbracket X, Y \rrbracket_{i}\right\rangle \in$ $H, r \notin Y$. In a similar way, $\mathbf{1}_{\text {give }}, \mathbf{1}_{\text {own }}$ and $\mathbf{1}_{\text {goal }}$ are defined. The intuition is that given a dialogue $H$ a proposal prop is more informative if its elements were not stated in previous locutions.

Suppose that $A g_{1}$ is the agent that starts the negotiation. For the sake of example, we summarize next the main steps in the first two moves in the negotiation process:

Move 1: $A g_{1}$ uses the algorithm Init $_{1}$ to compute the first proposal. The functions $\operatorname{Gen}\left(R_{1},\{\right.$ nail $\},\{$ hangPict $\left.\}, 1\right)$ and $\operatorname{Gen}\left(\{\right.$ nail $\}, R_{1},\{$ screw $\left.\}, 2\right)$ are computed, obtaining as a result

$$
\begin{aligned}
p \mathcal{S}_{1}= & \left\{\left\langle(\emptyset,\{\text { hangPict }\}), \llbracket\{\text { hangPict }\}, R_{1} \rrbracket_{1}\right\rangle,\right. \\
& \langle(\{\text { nail } \wedge \text { hammer } \rightarrow \text { hangpicture }, \\
& \text { hammer } \left.\left.\},\{\text { hangPict }\}), \llbracket\{\text { nail }\}, \emptyset \rrbracket_{1}\right\rangle, \ldots\right\}, \\
p \mathcal{B}_{1} \mathcal{S}_{2}= & \left\{\left\langle(\{\text { screw }\}, \emptyset), \llbracket \emptyset,\{\text { screw }\} \rrbracket_{1}\right\rangle\right\} .
\end{aligned}
$$

Now $A g_{1}$ can compute the potential deals from the set of its proposals (i.e., prop $\in p \mathcal{S}_{1}$ ) considering those it believes are solutions for $A g_{2}$ (i.e., $\odot($ prop $) \in$ $\left.\bigodot\left(p \mathcal{B}_{1} \mathcal{S}_{2}\right)\right)$ :

$$
\begin{aligned}
p \mathcal{B}_{1} \mathcal{D}= & \left\{\left\langle(\emptyset,\{\text { hangPict }\}), \llbracket\{\text { hangPict }\},\{\text { screw }\} \rrbracket_{1}\right\rangle\right. \\
& \langle(\{\text { hammer }, \text { nail } \wedge \text { hammer } \rightarrow \text { hangPict }\}, \\
& \left.\left.\{\text { hangPict }\}), \llbracket\{\text { nail }\},\{\text { screw }\} \rrbracket_{1}\right\rangle\right\} \\
= & \{\text { prop } 1, \text { prop } 2\} .
\end{aligned}
$$

Since this is the first move, $H_{1}$ is empty and thus propSet $=p \mathcal{B}_{i} \mathcal{D}$. Then the select function, which maximizes a weight sum, must choose between prop 1 and prop 2 . Note that the first proposal is less informative and the resources allocation is less balanced than the second proposal, computing $U($ prop 1$)=8$, $U($ prop 2$)=0, I($ prop 1$)=3, U($ prop 2$)=5$, therefore $\lambda_{U} U($ prop 1$)+\lambda_{I} I($ prop 1$)=8$, and $\lambda_{U} U($ prop 2$)+\lambda_{I} I($ prop 2$)=10$. Therefore the select function chooses the second proposal, adding it to $H_{1}$ and $A g_{1}$ is ready to start the negotiation with the following proposal:

I propose that you provide me with nail, because if I use hammer and nail $\wedge$ hammer $\rightarrow$ hangPict, then I can achieve hangPict in exchange for screw. 
Move 2: $A g_{2}$ receives $A g_{1}$ 's proposal, and invokes the Answer $_{2}$ algorithm. $A g_{2}$ adds the proposal to $\mathrm{H}_{2}$ and then uses the Gen function to compute $p \mathcal{S}_{2}$ :

$$
\begin{aligned}
p \mathcal{S}_{2} & =\operatorname{Gen}\left(R_{2}, \emptyset,\{\text { hang } M i r\}, 2\right) \\
& =\left\{\left\langle(\{\text { hammer }\}, \emptyset), \llbracket R_{2},\{\text { hangMir }\} \rrbracket_{2}\right\rangle\right\} .
\end{aligned}
$$

Since $\odot($ prop $) \notin \odot\left(p \mathcal{S}_{2}\right)$ (that is to say, $\llbracket\{$ screw $\},\{$ nail $\} \rrbracket_{2} \notin\left\{\llbracket\{\right.$ hammer $\left.\left.\}, \emptyset \rrbracket_{2}\right\}\right), A g_{2}$ does not accept, using the proposal information to update its beliefs, and its Init $_{2}$ function to generate a proposal to answer $A g_{1}$. The current mental state of $A g_{2}$ is now as follows:

$$
\begin{aligned}
R_{2}= & \{\text { nail, screwDriver, hammer } \wedge \text { nail } \rightarrow \\
& \text { hangMir }\}, \\
G_{2}= & \{\text { hangMir }\}, \\
B_{2} R_{1}= & \{\text { screw, hammer, nail } \wedge \text { hammer } \rightarrow \\
& \text { hangPict }\}, \\
B_{2} G_{1}= & \{\text { hangPict }\}, \\
H_{2}= & {[\langle(\{\text { hammer, nail } \wedge \text { hammer } \rightarrow} \\
& \text { hangPict }\},\{\text { hangPict }\},\{\text { screw }\}),\{ \\
& \text { nail }\}\rangle] .
\end{aligned}
$$

Notice that $A g_{2}$ through its interpretation process has revised its previous beliefs about $A g_{1}$ 's goal, and now it believes that its goal is hangPict.

The whole dialogue obtained in the negotiation program for this scenario is the following:

1 Says: I propose that you provide me with [nail], because if I use [hammer, nail\&hammer $=>$ hangPicture], then I can achieve [hangPicture] in exchange for [screw].

2 Says: I propose that you provide me with [hangMirror], because if I use [], then I can achieve [hangMirror] in exchange for [nail].

1 Says: I propose that you provide me with [nail], because if I use [hammer, nail\&hammer $=>$ hangPicture], then I can achieve [hangPicture] in exchange for [screw, screwDriver\&screw $=>$ hangMirror].

2 Says: accept. I give you [nail] and you give me [screw, screwDriver\&screw $=>$ hangMirror].

\section{Simulations}

In order to assess the benefits of using belief revision in the context of our proposal for argument-based negotiation, simulations of bilateral negotiation were carried out, considering different scenarios. In each case, agents had to cope with missing and wrong beliefs about their counterpart.
Generating the scenarios. The assessment of our proposal was based on 100 randomly generated negotiation scenarios. The process for generating a scenario is based on randomly selecting the goals for each agent $G_{1}, G_{2} \in G_{\mathcal{L}}$, generating then three disjoint sets of resources $F, S, T \in R_{\mathcal{L}}$ such that $F \vdash G_{1}, S \vdash G_{2}$ and $T \vdash G_{1} \wedge G_{2}$. Then the mental state for $A g_{1}$ and $A g_{2}$ was defined as $M S_{1}=\left\langle R_{1}, G_{1}, B_{1} R_{2}, B_{1} G_{2}, H_{1}\right\rangle$ and $M S_{2}=\left\langle R_{2}, G_{2}, B_{2} R_{1}, B_{2} G_{1}, H_{2}\right\rangle$ such that

(i) $R_{1}=F_{1} \cup S_{1} \cup T_{1}, \quad R_{2}=F_{2} \cup S_{2} \cup T_{2}$, where $F_{1}, F_{2}$ (resp., $S_{1}, S_{2}$, and $T_{1}, T_{2}$ ) are partitions of $F$ (resp., $S$ and $T$ );

(ii) $B_{1} R_{2} \subset R_{2} \cup R_{1}, \quad B_{2} R_{1} \subset R_{1} \cup R_{2}$;

(iii) $B_{1} G_{2}=G_{1}, \quad B_{2} G_{1}=G_{2}$;

(iv) $H_{1}=H_{2}=\langle\rangle$.

We can see that $F=F_{1} \cup F_{2}$ is a solution for $A g_{1}, S=$ $S_{1} \cup S_{2}$ is a solution for $A g_{2}$, and $T=T_{1} \cup T_{2}$ can be a solution for both agents. With this allocation of resources and the agent's beliefs, we ensure that initially each agent cannot achieve its own goal by itself and both agents have incomplete and wrong beliefs about their counterpart.

After creating these negotiation scenarios, three different types of agents were distinguished, based on the characterization of their decision making apparatus. In each case, a particular definition of the Answer function was considered, characterizing

1. NBR agents: these agents do not use belief revision techniques for argument generation and interpretation;

2. PBR agents: these agents implement belief revision using only the information contained in the argument claim (proposal);

3. BR agents: these agents take advantage of belief revision using all the information contained in the argument (i.e., proposal and support). This corresponds to the full-fledged version of our proposal.

It must be noted that these different types of agents (NBR, PBR, BR) share the same underlying structure and the only difference among them is associated with the role of the belief revision process during the negotiation (i.e., no belief revision for NBR agents, applying belief revision using only the proposal for PBR agents, and applying belief revision considering both proposal and support for BR agents). In order to implement these different characterizations, we will modify part of the code of Algorithm 2, as discussed below.

We ran simulations using two negotiating agents of the same type (i.e., NBR, PBR and BR) in the different 
negotiating scenarios which were randomly generated (100 cases). In all the simulations, both agents used the selection function described previously, i.e., the agents select the proposal prop $\in$ Proposal that maximizes a weighted sum $\lambda_{U} U($ prop $)+\lambda_{I} I($ prop $)$, using a balanced approach that weights equally the informativeness of the proposal and its associated utility, i.e., $\lambda_{U}=\lambda_{I}=0.5$. Besides, in all the negotiation scenarios it was assumed that $A g_{1}$ starts the negotiation dialogue.

In each simulation we analyzed (i) whether there was an agreement in the negotiation (i.e., it finished with accept or withdraw) and (ii) the length of the negotiation process (i.e., the number of iterations). Besides, we were interested in assessing the evolution of the agent's beliefs with respect to its initial mental state. In order to do this, we analyzed two ratios: on the one hand, for each scenario we evaluated the decrease in the agent's missing and wrong beliefs (see Definition2). We computed the ratio of these two kinds of beliefs an agent has at the end of negotiation with respect to the initial ones it had as follows:

$$
\frac{\left|M^{\text {end }}\right|+\left|F^{\text {end }}\right|}{\left|M^{\text {init }}\right|+\left|F^{\text {init }}\right|} .
$$

On the other hand, for each case we compute how the correct beliefs increase during the negotiation process. This is computed as

$$
\frac{\left|T^{e n d}\right|}{\left|T^{\text {init }}\right|} .
$$

Simulations of NBR agents. We modeled the decision making apparatus of the agents $A g_{1}$ and $A g_{2}$ using the proposed algorithms (i.e., Init and Answer) but without considering the belief revision process. To proceed accordingly, Algorithm 2 (Section 4.4) was modified, eliminating Lines 6 to 9 . The outcomes of the 100 negotiations with agents modeled as NBR agents are shown in Fig. 5. We can observe that there is an agreement in only $15 \%$ of the negotiation cases, with an average of negotiation length of 35 (i.e., the number of messages exchanged). On the other hand, $85 \%$ of the negotiations finished without an agreement (i.e., ending with a withdraw). Since the agents do not implement a belief revision process during the negotiation, their beliefs about their opponent remain unchanged (i.e., their missing and wrong beliefs wrt their counterpart's resources are not modified during the negotiation process).

Simulations using PBR agents. For these simulations we modeled the agent's decision making apparatus by restricting the belief revision process only on the arguments claim (without considering it for proposal justification). To achieve this, we modified Algorithm 2 eliminating Lines 7 to 9. In Fig. 6 we show the output of

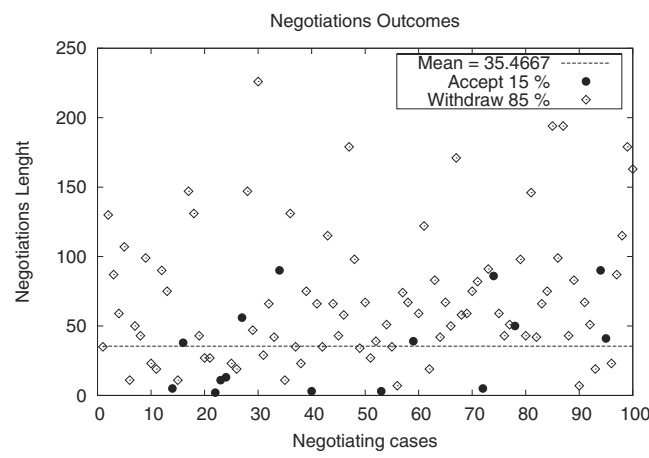

Fig. 5. Output of negotiations with NBR agents.

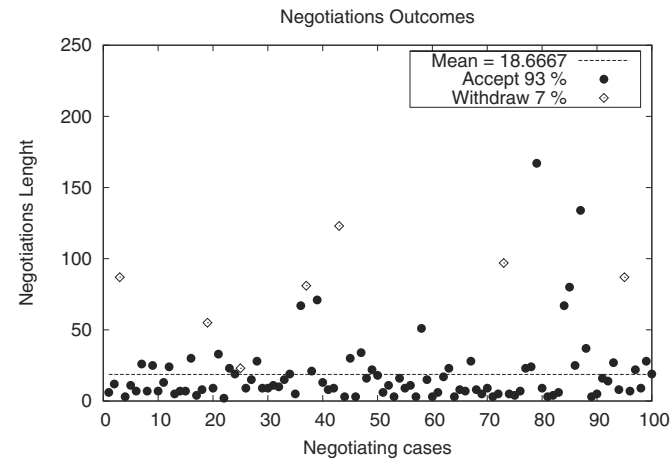

Fig. 6. Output of negotiations with PBR agents.

negotiations using PBR agents. We can observe that the agents reached an agreement in $93 \%$ of the negotiations, whereas only in $7 \%$ they did not. Moreover, in Fig. 7(a) we can see the percentage of reduction of missing and wrong beliefs for $A g_{1}$ about its counterpart, and in Fig. 7(b) the increase in correct beliefs for $A g_{1}$ about its opponent. We can see that a PBR agent reduces to an average of $57 \%$ its missing and wrong beliefs about its counterpart. On the other hand, its correct beliefs about its opponent resources increase an average of $178 \%$ until the agent reaches an agreement.

Simulations of BR agents. Finally, we will analyze the simulations using the full-fledged proposed agent negotiation model (BR agents). The output is shown in Fig. 8, where an agreement was reached in $96 \%$ of the cases, whereas only $4 \%$ ended with a withdraw.

Figure 9a) shows the reduction in missing and wrong beliefs for $A g_{1}$, and Fig. 9(b) illustrates the percentage of the increase in its correct beliefs (knowledge acquisition) wrt its counterpart's resources. It can be observed that in reaching an agreement the BR agent reduced on average $60 \%$ of its missing and wrong beliefs with respect to $A g_{2}$ and increased on average $176 \%$ of 


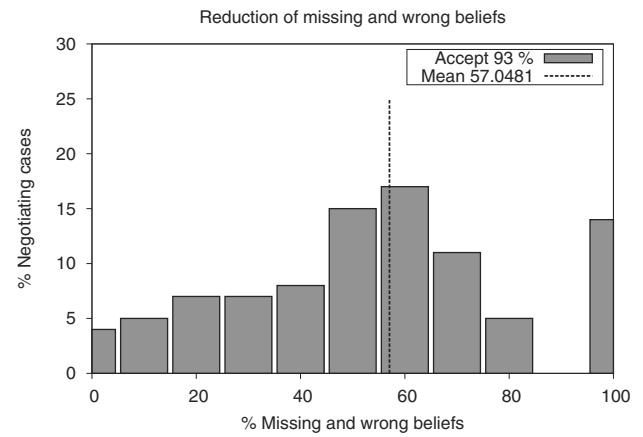

(a)

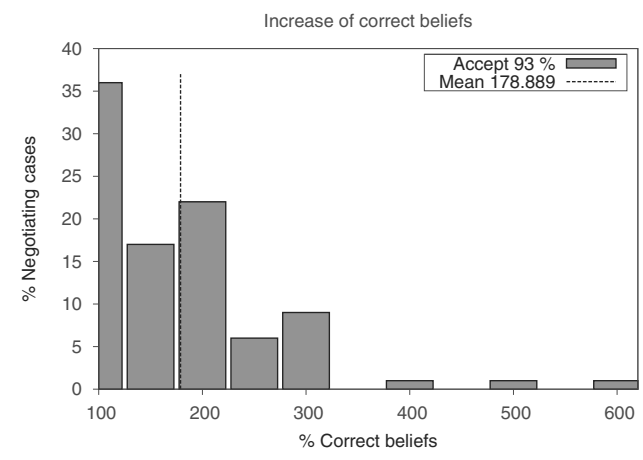

(b)

Fig. 7. PBR agents: reduction in missing and wrong beliefs (a), acquired knowledge (b).

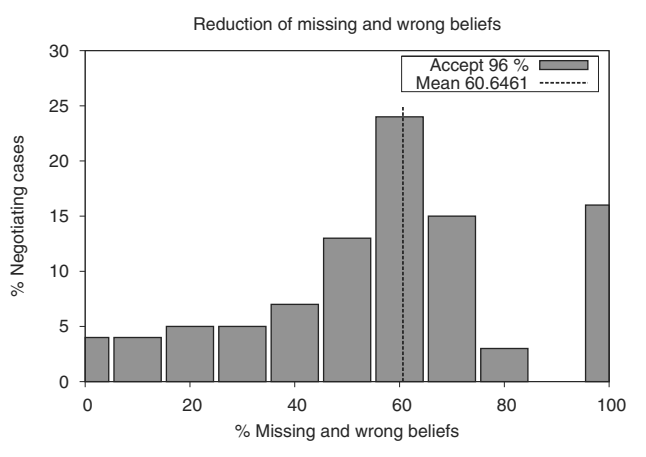

(a)

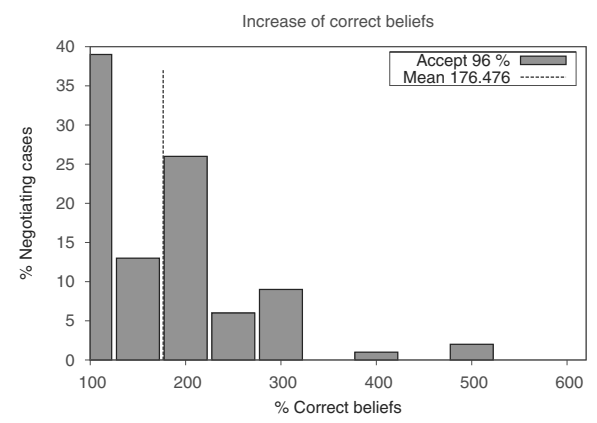

(b)

Fig. 9. BR agents: reduction in missing and wrong beliefs (a), acquired knowledge (b).

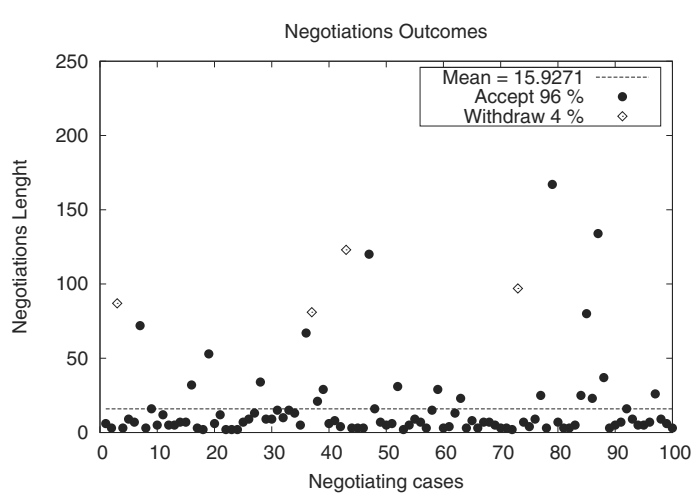

Fig. 8. Output of negotiations with BR agents.

its correct beliefs on its counterpart resources when the negotiation finished.

These simulations allow assessing the impact of belief revision on the negotiation process. On the one hand, agents that implemented belief revision (PBR and
BR agents) reached agreements in many more cases (93\% and $96 \%$ ) than NBR agents, which do not use revision $(15 \%)$. On the other hand, the negotiation length tends to be shorter in those agents that take advantage of belief revision (as the average number of iterations decreased from 35.47 in NBR agents to 18.67 in PBR agents, and the smallest average of 15.93 was obtained for BR agents). Concerning the reduction of missing and wrong beliefs, PBR agents had an average of $57.05 \%$, a slightly lower value than the one for BR agents $(60.65 \%)$. Besides, the average of increase for correct beliefs is $178.89 \%$ for PBR agents and $176.48 \%$ for BR agents (i.e., percentages which are very similar). BR agents they have achieved agreements in more negotiation cases and faster than PBR agents. However, they end the negotiation having on average slightly more missing and wrong beliefs and less correct beliefs than PBR agents. Intuitively, BR Agents are able to reach an agreement under more incomplete or wrong beliefs. Finally, we can observe that there is a considerable difference in the negotiation results (i.e., the number of agreements and length) between agents that incorporate belief revision as part of their decision making machinery and those which do not. 


\section{Related work}

In contrast with the original argumentative framework to solve the HIA problem (Parsons et al., 1998), our negotiation model allows the agents to gain and revise their beliefs as the dialogue takes place. Consequently, in our approach an agent does not need to have initial (or correct) beliefs about the other agent involved in the negotiation. In the works of Rahwan et al. (2007) and Pasquier et al. (2011), a similar scenario is analyzed, but agents are aware of all the agents' resources, and the agents' plans (or their knowledge about plans) are not consider negotiable. We think that our proposal is more flexible in this respect, as plans are also negotiation objects in our formalization.

There have been previous approaches integrating belief revision and negotiation. In the work of Zhang et al. (2004) a formal characterization of negotiation from a belief revision perspective is given, but no implementation issues are considered. Additionally, it must be noted that in our proposal we assume that agents are benevolent. This approach can also be found in several frameworks (e.g., Parsons et al., 1998; Amgoud et al., 2000). In addition, in our work, agents are assumed to be truthful. Recent research has led to considering other situations such as negotiation among dishonest agents (Sakama, 2011), which is an interesting scenario for future work. Another relevant approach to argumentation-based negotiation can be seen in the work of Amgoud et al. (2007), where the proposed framework makes it possible to study the outcomes of the negotiation process. In contrast to this approach, our proposal relies on the characterization of belief revision operations to model agents' arguments generation, which their claims are the resources to be exchanged.

Formal models of belief change can be very helpful in providing suitable frameworks for rational agents (Bonanno et al., 2009), in which the information from inter-agent dialogues can be better exploited. Part of our recent research work (Pilotti et al., 2014) includes adding a justification when a proposal is rejected. However, no empirical analysis of this model has been carried out yet.

Inspired in human negotiation procedures, Sierra and Debenham (2007) consider five dimensions relevant to successful negotiation: legitimacy, options, goals, independence, and commitment. They introduce a negotiation model based on these dimensions and two primitive concepts: intimacy (degree of closeness) and balance (degree of fairness). As the agents representing their human principals may not be just utility maximizers, in some cases they aim at building long lasting relationships with progressing levels of intimacy that determine what balance in information and resource sharing is acceptable to them. These two concepts, intimacy and balance, are key to understanding competitive and co-operative game theory as two particular theories of agent relationships (i.e., at different intimacy levels).

\section{Conclusions}

In this paper we presented a novel approach to automated negotiation between two argumentative agents. An intentional architecture was given to each agent as to represent not only its own resources and goals but also its beliefs about the other agent's resources and goals. In our approach, the interpretation and generation of arguments are based on belief revision operators. In order to achieve their goals, agents engage in a benevolent dialogue, exchanging information that supports which resources they are willing to exchange. During the negotiation, the agents continuously update their mental states to generate proposals more likely to be accepted. All the propositions presented in our approach were formalized in Coq. A revised version of HIA was solved, showing how the agents can negotiate to solve this kind of cooperative problem, starting with incomplete and wrong beliefs about the other agent's resources, plans and goals. As discussed in Section 6, we carried out an empirical analysis of our proposal, assessing the impact of considering belief revision during the negotiation process.

Part of our future work is focused on studying complexity issues related to our proposal, as done by Zhang (2010) in the context of belief-revision based bargaining and negotiation. Furthermore, we want to identify different kinds of negotiation problems for which either BR or PBR agents are to be preferred, considering the trade-off between negotiation results and computational complexity. We are also investigating the logical properties of our approach, as well as the impact of different cost assignments in our model. In this setting, we contend that extending the integration of argumentation and belief revision in the context of agent negotiation dialogues is a very promising area for future research.

\section{Acknowledgment}

This research work was partially supported by the research projects PICT-ANPCyT 2009-0015, PID-UNR ING 466, PIP-Conicet 112-200801-02798 and the LACCIR project R1211LAC004.

\section{References}

Alchourrón, C., Gärdenfors, P. and Makinson, D. (1985) On the logic of theory change: Partial meet contraction and revision functions, Journal of Symbolic Logic 50(2): 510-530.

Amgoud, L., Dimopoulos, Y. and Moraitis, P. (2007). A unified and general framework for argumentation-based 
negotiation, 6th International Joint Conference on Autonomous Agents and Multiagent Systems (AAMAS 2007), Honolulu, HI, USA, p. 158.

Amgoud, L., Parsons, S. and Maudet, N. (2000). Arguments, dialogue and negotiation, in W. Horn (Ed.), Proceedings of the European Conference on Artificial Intelligence (ECAI 2000), IOS Press, Berlin, pp. 338-342.

Amgoud, L. and Vesic, S. (2011). A formal analysis of the outcomes of argumentation-based negotiations, 10th International Conference on Autonomous Agents and Multiagent Systems (AAMAS 2011), Taipei, Taiwan, pp. 1237-1238.

Bonanno, G., Delgrande, J., Lang, J. and Rott, H. (2009). Special issue on formal models of belief change in rational agents, Journal of Applied Logic 7(4): 363.

Dix, J., Hansson, S.O., Kern-Isberner, G. and Simari, G.R. (2013). Belief change and argumentation in multi-agent scenarios (Dagstuhl seminar 13231), Dagstuhl Reports 3(6): 1-21.

Dung, P.M. (1995). On the acceptability of arguments and its fundamental role in nonmonotonic reasoning, logic programming and n-person games, Artificial Intelligence 77(2): 321-357.

Falappa, M., Garcia, A., Kern-Isberner, G. and Simari, G. (2011). On the evolving relation between belief revision and argumentation, Knowledge Engineering Review 26(1): 35-43.

Fermé, E., Saez, K. and Sanz, P. (2003). Multiple kernel contraction, Studia Logica: An International Journal for Symbolic Logic 73(2): 183-195.

Hansson, S. (1994). Kernel contraction, The Journal of Symbolic Logic 59(3): 845-859.

Hansson, S. (1999). A Textbook of Belief Dynamics: Theory Change and Database Updating, Applied Logic Series, Kluwer Academic Publishers, Dordrecht.

Jennings, N.R., Faratin, P., Lomuscio, A.R., Parsons, S., Sierra, C. and Wooldridge, M. (2001). Automated negotiation: Prospects, methods and challenges, International Journal of Group Decision and Negotiation 10(2): 199-215.

Kraus, S., Sycara, K. and Evenchik, A. (1998). Reaching agreements through argumentation: A logical model and implementation, Artificial Intelligence 104(1-2): 1-69.

Parsons, S., Sierra, C. and Jennings, N.R. (1998). Agents that reason and negotiate by arguing, Journal of Logic and Computation 8(3): 261-292.

Pasquier, P., Hollands, R., Rahwan, I., Dignum, F. and Sonenberg, L. (2011). An empirical study of interest-based negotiation, Autonomous Agents and Multi-Agent Systems 22(2): 249-288.

Pilotti, P., Casali, A. and Chesñevar, C. (2014). Incorporating object features in collaborative argumentation-based negotiation agents, Brazilian Conference on Intelligent Systems (BRACIS)/Encontro Nacional de Inteligencia Artificial e Computacional (ENIAC), Sao Carlos, SP, Brazil, pp. 31-37.
Rahwan, I., Pasquier, P., Sonenberg, L. and Dignum, F. (2007). On the benefits of exploiting underlying goals in argument-based negotiation, 22nd Conference on Artificial Intelligence (AAAI), Vancouver, Canada, pp. 116-121.

Rahwan, I., Ramchurn, S.D., Jennings, N.R., Mcburney, P., Parsons, S. and Sonenberg, L. (2003). Argumentation-based negotiation, Knowledge Engineering Review 18(4): 343-375.

Ramchurn, S.D., Jennings, N.R. and Sierra, C. (2003). Persuasive negotiation for autonomous agents: A rhetorical approach, Proceedings of the IJCAI Workshop on Computational Models of Natural Argument, Acapulco, Mexico, pp. 9-17.

Rosenschein, J.S. and Zlotkin, G. (1994). Rules of Encounter: Designing Conventions for Automated Negotiation among Computers, MIT Press, Cambridge, MA.

Sadri, F., Toni, F. and Torroni, P. (2001). Logic agents, dialogues and negotiation: An abductive approach, Proceedings of the AISB'01 Convention, York, UK, pp. 5-12.

Sakama, C. (2011). Dishonest reasoning by abduction, 22nd International Joint Conference on Artificial Intelligence, Barcelona, Spain, pp. 1063-1064.

Sierra, C. and Debenham, J.K. (2007). The logic negotiation model, 6th International Joint Conference on Autonomous Agents and Multiagent Systems (AAMAS 2007), Honolulu, HI, USA, p. 243.

Zhang, D. (2010). A logic-based axiomatic model of bargaining, Artificial Intelligence 174(16-17): 1307-1322.

Zhang, D., Foo, N., Meyer, T. and Kwok, R. (2004). Negotiation as mutual belief revision, Proceedings of AAAI04, San Jose, CA, USA, pp. 317-322.

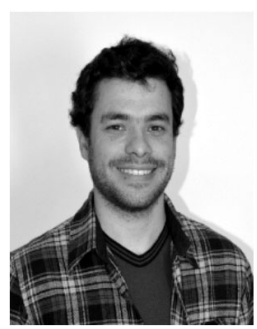

Pablo Pilotti has a degree in computer science from Universidad Nacional de Rosario (UNR), Argentina. He holds a teaching position in artificial intelligence at the Faculty of Exact Sciences, Engineering and Land Surveying (FCEIAUNR) in the Department of Computer Science. He currently is a Ph.D. student at the French Argentine International Center for Information and Systems Sciences (CIFASIS) under the direction of Dr. Ana Casali and Dr. Carlos Chesñevar.

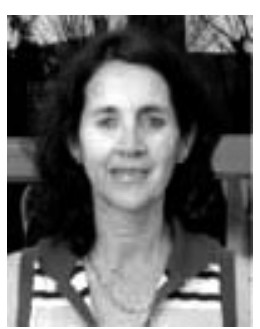

Ana Casali received her Ph.D. and Master's degrees in information technologies at the University of Girona, Spain, and a degree in mathematics at Universidad Nacional de Rosario (UNR), Argentina. She has been the head of the Computer Science Department since 2007 and a professor at Facultad de Ciencias Exactas, Ingeniera y Agrimensura, UNR, since 1991. Also, she is a researcher at the French Argentine International Center for Information and Systems Sciences (CIFASIS). She has worked in several international cooperation research projects, and her research interests include agent architectures, knowledge representation, approximate reasoning, recommender systems and its applications to education. She also is the author of many articles, mainly in the field of artificial intelligence and its applications. 


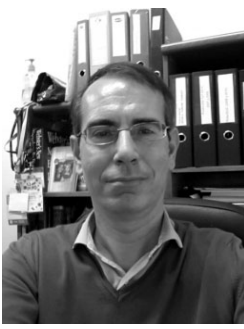

Carlos Chesñevar is an independent researcher from the National Council of Scientific and Technical Research (CONICET) of Argentina since 2004, and an associate professor at the Department of Computer Science and Engineering of Universidad Nacional del Sur (Baha Blanca, Argentina). He has carried out research in various universities (Imperial College, London, UK; University Leipzig, Germany; Washington University, USA, and others). He has supervised several $\mathrm{Ph} . \mathrm{D}$. and M.Sc. theses in computer science. He has participated as a PC member in most major AI conferences (IJCAI, AAMAS, ECSQARU, and others). He has published more than 20 journal articles, 7 book chapters and over 100 papers in conference proceedings.

Received: 7 July 2014

Revised: 15 November 2014 